\title{
A COLLOCATION METHOD FOR A CLASS OF NONLINEAR SINGULAR INTEGRAL EQUATIONS WITH A CARLEMAN SHIFT
}

\author{
Mohamed M. Allan \\ Department of Mathematics, Faculty of Science, Zagazig University, Zagazig, Egypt \\ m_m_allan@hotmail.com
}

\begin{abstract}
The paper is concerned with the applicability of the collocation method to a class of nonlinear singular integral equations with a Carleman shift preserving orientation on simple closed smooth Jordan curve in the generalized Holder space $H_{\varphi}(L)$.
\end{abstract}

Key Words: Nonlinear singular integral equations, Carleman shift, Collocation method, Noether operator.

\section{INTRODUCTION}

Nonlinear singular integral equations are widely used and connected with applications in several field of engineering mechanics like structural analysis, fluid mechanics and aerodynamics. This leads to the necessity to derive solutions for the nonlinear singular integral equations arising in applications, by using some approximate and constructive methods, (see [16] ).

The theory of nonlinear singular integral equations with Hilbert and Cauchy kernel and its related Riemann-Hilbert problems have been developed in works of Pogorozelski W. [20], Guseinov A. I and Mukhtarove Kh. Sh. [9], Wolfersdorf L.V. [24], Ladopoulous E.G [16] and others.

The successful development of the theory of singular integral equations (SIE) naturally stimulated the study of singular integral equations with shift (SIES). The Noether theory of singular integral operators with shift (SIOS) is developed for a closed and open contour (see [12-14], 17] and others).

Existence results and approximate solutions have been studied for nonlinear singular integral equations (NSIE) and nonlinear singular integral equations with shift (NSIES) by many authors among them we mention (1-6, 10,11,15,16,19,21).

The classical and more recent results on the solvability of NSIE should be generalized to corresponding equations with shift, (see[23]). The theory of SIES is an important part of integral equations because of its recent applications in many field of physics and engineering,(see $[7,13,14])$

We consider a simple closed smooth Jordan curve $L$ in the complex plane with equation $t=t(s), 0 \leq s \leq l$ where s-arc coordinate accounts from some fixed point, $l$ length of the curve $L$. Denote by $D^{+}$and $D^{-}$the interior and exterior domain of $L$ respectively and let the origin be $0 \in D^{+}$. Denote by $L_{0}$ the unite circle with the center at the origin and let $L_{0}{ }^{+}$and $L_{0}{ }^{-}$the interior and exterior domain of $L_{0}$ respectively. 
Consider the conformal mappings $A(r)$ from $L_{0}{ }^{-}$onto $D^{-}$such that $A(\infty)=\infty$, $\lim _{r \rightarrow \infty} A(r) r^{-1}>0$ and $B(r)$ from $L_{0}{ }^{-}$onto $D^{+}$such that $B(\infty)=0$.

Now, consider the following NSIES:

$$
\begin{aligned}
(\mathrm{P}(u))(t)=\Psi_{1}(t, u(t))+ & \Psi_{2}(\alpha(t), u(\alpha(t)))- \\
& -\frac{1}{\pi i} \int_{L}\left[\frac{\Psi_{3}(\tau, u(\tau))}{\tau-t}+\frac{\Psi_{4}(\tau, u(\tau))}{\tau-\alpha(t)}\right] d \tau=f(t), \text { forall } t \in L
\end{aligned}
$$

Under the following conditions

$$
\psi_{1 u}\left(t, u_{o}(t)\right)=\psi_{3 u}\left(t, u_{o}(t)\right)=a(t), \quad \psi_{2 u}\left(\alpha(t), u_{o}(\alpha(t))\right)=-\psi_{4 u}\left(\alpha(t), u_{o}(\alpha(t))\right)=b(t) .
$$

for initial value $u_{0}$, in the generalized Holder space $H_{\varphi}(L), u(t)$ is unknown function, $f(t)$ and $\Psi_{r}(t, u(t)), r=1, \ldots, 4$, are continuous functions on $L$ and on the domain

$$
D=\{(t, u): t \in L, u \in(-\infty, \infty)\},
$$

respectively. and the homeomorphism $\alpha: L \rightarrow L$ is preserving orientation, satisfying the Carleman condition

$$
\alpha(\alpha(t))=\alpha_{2}(t)=t, \quad t \in L,
$$

and the derivative $\alpha^{\prime}(t) \neq 0$ satisfies the usual Holder condition.

The equation (1.1) in case $f(t)=0$, without shift has been studied in [4] by modified Newton-Kantorovich method in the generalized Holder space $H_{\varphi, m}[a, b]$.

In this paper the polynomial collocation method has been applied to NSIES (1.1) under condition (1.2), with zero index, in the generalized Holder space $H_{\varphi}(L)$.

\section{SOME AUXILIARY RESULTS}

Definition 2.1. We denote by $H_{\varphi, 1}(D)$ to be the space of all functions $\Psi_{r}(t, u(t)), r=1, \ldots, 4$, which have partial derivatives up to second order with respect to $u$ and satisfy the following condition

$$
\left|\psi_{r u^{j}}\left(t_{1}, u_{1}\right)-\psi_{r u^{j}}\left(t_{2}, u_{2}\right)\right| \leq c_{j}^{r}\left\{\varphi\left(\left|t_{1}-t_{2}\right|\right)+\left|u_{1}-u_{2}\right|\right\},
$$

where $\left(t_{i}, u_{i}\right) \in D, i=1,2, \varphi \in \Phi$ and $c_{j}^{r}$ are constants; $j=0,1,2$.

Definition $2.2[12,18]$. We denote by $c(L)$ the space of all continuous functions $u(t)$ defined on $L$ with the norm:

$$
\|u\|_{c(L)}=\max _{t \in L}|u(t)| \text {. }
$$

Definition 2.3 [5,9]. We denote by $H_{\varphi}(L)$ the space of all functions $u(t) \in c(L)$ such that $\omega_{u}(\delta)=o(\varphi(\delta)), \varphi \in H \Phi$, with the norm:

$$
\begin{gathered}
\|u\|_{\varphi}=\|u\|_{c(L)}+\|u\| \\
\|u\|=\sup _{\delta>0} \frac{\omega_{u}(\delta)}{\varphi(\delta)} ;
\end{gathered}
$$


$H \Phi=\left\{\varphi \in \Phi: \int_{0}^{\delta} \frac{\varphi(\xi)}{\xi} d \xi+\delta \int_{\delta}^{l} \frac{\varphi(\xi)}{\xi^{2}} d \xi \leq \tilde{c} \varphi(\delta)\right\}$,

$\widetilde{c}$ is a positive constant.

Definition $2.4[5,14]$. Let $S: H_{\varphi}(L) \rightarrow H_{\varphi}(L)$ denotes to the operator of singular integration

$$
(S u)(t)=\frac{1}{\pi i} \int_{L} \frac{u(\tau)}{\tau-t} d \tau,
$$

to which we associate the Cauchy projection operators

$$
P_{ \pm}=\frac{1}{2}(I \pm S), \quad S^{2}=I,
$$

where I is the identity operator on $H_{\varphi}(L)$. The Carleman shift operator

$$
W: H_{\varphi}(L) \rightarrow H_{\varphi}(L),
$$

is given by $(W v)(t)=v(\alpha(t))$.

Lemma 2.1 [5]. The singular operator $S$ is abounded operator on the space $H_{\varphi}(L)$ and satisfies the inequality

$$
\|S u\|_{\varphi} \leq \rho_{0}\|u\|_{\varphi},
$$

where $\rho_{0}$ is a constant defined as follows :

$$
\rho_{0}=c_{1}\left(\int_{0}^{\delta} \frac{\varphi(\xi)}{\xi} d \xi+1\right)+c_{2} \tilde{c},
$$

where $c_{1}, c_{2}, \tilde{c}$ are constants.

Lemma 2.2 [5]. The shift operator $W$ is a linear bounded continuously invertible operator on the space $H_{\varphi}(L)$ and satisfies the inequality

$$
\|W u\|_{\varphi} \leq \gamma_{0}\|u\|_{\varphi},
$$

where $\gamma_{0}=\max \left\{1, \alpha_{0}\right\}$ and $\alpha_{0}$ is a constant given by $\alpha_{0}=\sup _{\delta \succ 0} \frac{\omega_{\tilde{u}}(\delta)}{\omega_{u}(\delta)}, \tilde{u}(t)=u(\alpha(t))$.

Lemma 2.3 [1] Let the functions $\psi_{r}(t, u), r=1, \ldots, 4$, belong to $H_{\varphi, 1}(D)$. Then the operator $\mathrm{P}(u)$ is Frechet differentiable at every fixed point $u \in H_{\varphi}(L)$, moreover

$$
\begin{aligned}
\mathrm{P}^{\prime}(u) h=\psi_{1 u}(t, u(t)) h(t)+ & \psi_{2 u}(\alpha(t), u(\alpha(t))) h(\alpha(t))- \\
& -\frac{1}{\pi i} \int_{L}\left\{\frac{\psi_{3 u}(\tau, u(\tau))}{\tau-t}+\frac{\psi_{4 u}(\tau, u(\tau))}{\tau-\alpha(t)}\right\} h(\tau) d \tau,
\end{aligned}
$$

satisfies Lipschitz condition

$$
\left\|\mathrm{P}^{\prime}\left(u_{1}\right)-\mathrm{P}^{\prime}\left(u_{2}\right)\right\|_{\varphi} \leq \rho_{1}\left\|u_{1}-u_{2}\right\|_{\varphi},
$$


In the sphere $S_{\varphi}\left(u_{0}, r\right)=\left\{u \in H_{\varphi}(L):\left\|u-u_{0}\right\|_{\varphi} \leq r\right\}$, where

$$
\rho_{1}=\left(c_{1}^{1}+\gamma_{0} c_{1}^{2}+\rho_{0} c_{1}^{3}+\gamma_{0} \rho_{0} c_{1}^{4}\right) \text {. }
$$

Under condition (1.2) The equation (2.8) reduces to the following SIES, for the unknown function $h(t)$ :

$$
\begin{aligned}
\Gamma_{0} h=a(t) h(t)+b(t) h(\alpha(t))-\frac{a(t)}{\pi i} \int_{L} \frac{h(\tau)}{\tau-t} d \tau+\frac{b(t)}{\pi i} \int_{L} \frac{h(\tau)}{\tau-\alpha(t)} d \tau+ \\
\frac{1}{\pi i} \int_{L} R(t, \tau) h(\tau) d \tau=f(t),
\end{aligned}
$$

for initial value $u_{o}$ and the arbitrary function $f(t)$ belong to the space $H_{\varphi}(L)$, where

$$
R(t, \tau)=\frac{\psi_{3 u}\left(t, u_{o}(t)\right)-\psi_{3 u}\left(\tau, u_{o}(\tau)\right)}{\tau-t}+\frac{\psi_{4 u}\left(\alpha(t), u_{o}(\alpha(t))\right)-\psi_{4 u}\left(\tau, u_{o}(\tau)\right)}{\tau-\alpha(t)}
$$

Using Definition 2.4 the dominant equation of equation (2.10) reduces to the following singular integral operator with shift :

$$
M=2 a(t) P_{-}+2 b(t) W P_{+} .
$$

Theorem $2.1[1,5,14]$. The singular integral functional operator $M$ is Noetherian on $H_{\varphi}(L)$ if and only if

$$
\inf |e(t)|>0 \text { and } q(t) \neq 0 \text {, on } L \text {, }
$$

where

$$
e(t)=2 b(t), q(t)=\frac{a(t)}{b(t)} ; b(t) \neq 0 \text { on } L .
$$

The index of a Noetherian operator $M$ is given by

$$
\chi=\text { ind } M=\frac{1}{2 \pi}\{\arg q(t)\}_{L} .
$$

Theorem 2.2 [5,21]. Let the conditions of Lemma 2.3 and Theorem 2.1 be satisfied and $u_{0} \in H_{\varphi}(L)$ is the initial approximation for equation (1.1) under conditions (1.2), $\left\|\left(\mathrm{P}^{\prime}\left(u_{0}\right)\right)^{-1}\right\|_{\varphi} \leq \varepsilon_{0}$ and $\left\|\left(\mathrm{P}^{\prime}\left(u_{0}\right)\right)^{-1} \mathrm{P}\left(u_{0}\right)\right\|_{\varphi} \leq \varepsilon_{1}$. Then if $m=\varepsilon_{0} \rho_{1} \varepsilon_{1}<1 / 2$, then equation (1.1) under conditions (1.2) has a unique solution $u^{*}$ in the sphere $S_{\varphi}\left(u_{0} ; r_{0}\right)$ of the space $H_{\varphi}(L), r_{0}=\varepsilon_{1}(1-\sqrt{1-2 m}) m^{-1} \leq r$, to which the successive approximations: $u_{n+1}=u_{n}-\left(\mathrm{P}^{\prime}\left(u_{0}\right)\right)^{-1} \mathrm{P}\left(u_{n}\right)$ of modified Newton method converges and the rate of convergence is given by the inequality: $\left\|u_{n}-u^{*}\right\|_{\varphi} \leq \frac{B^{n}}{1-B} \varepsilon_{1} ; B=1-\sqrt{1-2 m}$ 


\section{COLLOCATION METHOD}

Now, we seek an approximate solution of equation (1.1) under conditions (1.2) in $H_{\varphi}(L)$ as the form:

$$
u_{n}(\eta, t)=\sum_{k=-n}^{n} \eta_{k} t^{k}
$$

where the coefficients $\eta_{k}$ are defined from the system of nonlinear algebraic equation with shift (SNAES)

$\Psi_{1}\left(t_{j}, u_{n}\left(\eta, t_{j}\right)\right)+\Psi_{2}\left(\alpha\left(t_{j}\right), u_{n}\left(\eta, \alpha\left(t_{j}\right)\right)\right)-\frac{1}{\pi i} \int_{L}\left[\frac{\Psi_{3}\left(\tau, u_{n}(\eta, \tau)\right)}{\tau-t_{j}}+\frac{\Psi_{4}\left(\tau, u_{n}(\eta, \tau)\right)}{\tau-\alpha\left(t_{j}\right)}\right] d \tau=f\left(t_{j}\right)$.

where $t_{j}=\exp (2 \pi i j /(2 n+1)), j=\overline{0,2 n}$.

Consider $(2 \mathrm{n}+1)$ - dimensional spaces $H_{\varphi}^{(1)}$ and $H_{\varphi}^{(2)}$ with the norms:

$$
\|\eta\|_{\varphi}^{(1)}=\left\|u_{n}(\eta, .)\right\|_{\varphi}, \quad\|u\|_{\varphi}^{(2)}=\max _{j}\left|u_{j}\right|+\sup _{j \neq k} \frac{\left|u_{j}-u_{k}\right|}{\varphi\left(\left|t_{j}-t_{k}\right|\right)},
$$

respectively, where $\eta=\left(\eta_{-n}, \ldots, \eta_{-1}, \eta_{0}, \ldots, \eta_{n}\right) \in H_{\varphi}^{(1)}$ and $u=\left(u_{0}, \ldots, u_{2 n}\right) \in H_{\varphi}^{(2)}$.

Introduce the operator $\mathrm{P}_{n}(\eta): H_{\varphi}^{(1)} \rightarrow H_{\varphi}^{(2)}$ where

$\mathrm{P}_{j, n}(\eta)=\Psi_{1}\left(t_{j}, u_{n}\left(\eta, t_{j}\right)\right)+\Psi_{2}\left(\alpha\left(t_{j}\right), u_{n}\left(\eta, \alpha\left(t_{j}\right)\right)\right)-$

$$
-\frac{1}{\pi i} \int_{L}\left[\frac{\Psi_{3}\left(\tau, u_{n}(\eta, \tau)\right)}{\tau-t_{j}}+\frac{\Psi_{4}\left(\tau, u_{n}(\eta, \tau)\right)}{\tau-\alpha\left(t_{j}\right)}\right] d \tau, \quad j=\overline{0,2 n}
$$

We can rewrite SNAES (3.2) in the operator form:

$$
\mathrm{P}_{n}(\eta)=f ; \quad f=f\left(t_{j}\right), j=\overline{0,2 n} .
$$

Consider, the coordinates of the vector $\eta^{(0)}$ from $H_{\varphi}^{(1)}$ these are the Fourier coefficients of the function $u_{0} \in H_{\varphi}(L)$ that is

$\eta_{j}^{(0)}=\frac{1}{2 \pi i} \int_{L_{0}} u_{0}(B(w)) w^{-j-1} d w, j=\overline{0, n}$ and $\eta_{j}^{(0)}=\frac{1}{2 \pi i} \int_{L_{0}} u_{0}(A(w)) w^{-j-1} d w, j=\overline{-n,-1}$

Analogous to Lemma 2.3 the following lemma is valid.

Lemma 3.1.[6] Let the conditions of Lemma 2.3 be satisfied. Then the operator $P_{n}$ is Frechet differentiable at every fixed point $x=\left(\eta_{-n}, \ldots, \eta_{n}\right) \in H_{\varphi}^{(1)}$, moreover

$$
\begin{aligned}
\mathrm{P}_{j, n}^{\prime}(x) h=\psi_{1 u}\left(t_{j}, u_{n}\left(x, t_{j}\right)\right) u_{n}\left(h, t_{j}\right)+\psi_{2 u}\left(\alpha\left(t_{j}\right), u_{n}\left(x, \alpha\left(t_{j}\right)\right)\right) u_{n}\left(h, \alpha\left(t_{j}\right)\right)- \\
-\frac{1}{\pi i} \int_{L}\left\{\frac{\psi_{3 u_{n}}\left(\tau, u_{n}(x, \tau)\right)}{\tau-t_{j}}+\frac{\psi_{4 u_{n}}\left(\tau, u_{n}(x, \tau)\right)}{\tau-\alpha\left(t_{j}\right)}\right\} u_{n}(h, \tau) d \tau, j=\overline{0,2 n .} \text { wh }
\end{aligned}
$$

ere $h=\left(h_{-n}, \ldots, h_{n}\right) \in H_{\varphi}^{(1)}$, the derivative $P_{n}^{\prime}(x)=\left(P_{0, n}^{\prime}(x), \ldots, P_{2 n, n}^{\prime}(x)\right)$ satisfies

Lipschitz condition 


$$
\left\|\mathrm{P}_{n}^{\prime}\left(x_{1}\right)-\mathrm{P}_{n}^{\prime}\left(x_{2}\right)\right\|_{H_{\varphi}^{(2)}} \leq \rho_{1}^{\prime}\left\|x_{1}-x_{2}\right\|_{H_{\varphi}^{(1)}},
$$

in the sphere $S\left(\eta^{(0)} ; r_{1}\right)$ of the space $H_{\varphi}^{(1)}$, where $\rho_{1}^{\prime}$ is a positive constant.

Now, we show that the system of linear algebraic equations with shift (SLAES):

under the conditions

$$
\mathrm{P}_{n}^{\prime}\left(\eta^{(0)}\right) h=g
$$

$$
\begin{gathered}
\psi_{1 u}\left(t_{j}, u_{o}\left(\eta^{(0)}, t_{j}\right)\right)=\psi_{3 u}\left(t_{j}, u_{o}\left(\eta^{(0)}, t_{j}\right)\right)=a\left(t_{j}\right), \\
\psi_{2 u}\left(\alpha\left(t_{j}\right), u_{o}\left(\eta^{(0)}, \alpha\left(t_{j}\right)\right)\right)=-\psi_{4 u}\left(\alpha\left(t_{j}\right), u_{o}\left(\eta^{(0)}, \alpha\left(t_{j}\right)\right)\right)=b\left(t_{j}\right) .
\end{gathered}
$$

has a unique solution $h \in H_{\varphi}^{(1)}$ for arbitrary $g=\left(g_{0}, \ldots, g_{2 n}\right) \in H_{\varphi}^{(2)}$.

For this aim, we consider the SALES:

$$
\begin{aligned}
\left.a\left(t_{j}\right) u_{n}\left(h, t_{j}\right)\right)+b\left(t_{j}\right) u_{n}\left(h, \alpha\left(t_{j}\right)\right)-\frac{a\left(t_{j}\right)}{\pi i} \int_{L} \frac{u_{n}(h, \tau)}{\tau-t_{j}} d \tau+\frac{b\left(t_{j}\right)}{\pi i} \int_{L} \frac{u_{n}(h, \tau)}{\tau-\alpha\left(t_{j}\right)} d \tau+ \\
+\frac{1}{\pi i} \int_{L} R\left(t_{j}, \tau\right) \mu_{n}(h, \tau) d \tau=g\left(t_{j}\right), j=\overline{0,2 n}
\end{aligned}
$$

corresponding to the SIES:

$$
\begin{aligned}
a(t) u(t)+b(t) u(\alpha(t))-\frac{a(t)}{\pi i} \int_{L} \frac{u(\tau)}{\tau-t} d \tau+\frac{b(t)}{\pi i} \int_{L} \frac{u(\tau)}{\tau-\alpha(t)} d \tau+ & \\
& +\frac{1}{\pi i} \int_{L} R(t, \tau) u(\tau) d \tau=g(t),
\end{aligned}
$$

According to the collocation method, we seek an approximate solution of equation (2.10) as the form :

$$
h_{n}(t)=\sum_{k=-n}^{n} \beta_{k} t^{k}, t \in L,
$$

where the coefficients $\beta_{k}$ are defined from SLAES:

$$
\sum_{k=-n}^{n} A_{j k} \beta_{k}=g\left(t_{j}\right), j=\overline{0,2 n}
$$

where

$$
A_{j k}=a\left(t_{j}\right)\left(t_{j}^{k}-\frac{1}{\pi i} \int_{L} \frac{\tau^{k}}{\tau-t_{j}} d \tau\right)+b\left(t_{j}\right)\left(\left(\alpha\left(t_{j}\right)\right)^{k}+\frac{1}{\pi i} \int_{L} \frac{\tau^{k}}{\tau-\alpha\left(t_{j}\right)} d \tau\right)+\frac{1}{\pi i} \int_{L} R\left(t_{j}, \tau\right) h_{n}(\tau) d \tau
$$

The SLAES (3.8) can be rewritten as following form:

$$
2 a\left(t_{j}\right) \sum_{k=-n}^{-1} \beta_{k} t_{j}^{k}+2 b\left(t_{j}\right) \sum_{k=0}^{n} \beta_{k}\left(\alpha\left(t_{j}\right)\right)^{k}+\frac{1}{\pi i} \int_{L} R\left(t_{j}, \tau\right) \sum_{k=-n}^{n} \beta_{k} \tau^{k} d \tau=g\left(t_{j}\right), j=\overline{0,2 n} \text {. }
$$

Where

$$
h_{n}^{+}(t)=\sum_{k=0}^{n} \beta_{k} t^{k}, h_{n}^{-}(t)=-\sum_{k=-n}^{-1} \beta_{k} t^{k}
$$


Theorem 3.1. Let $a(t), b(t)$ and $g(t)$ belong to $H_{\varphi}(L), b(t) \neq 0$ on $L$, the index $\chi=0$ and the operator $\mathrm{P}^{\prime}$ has a linear inverse in $H_{\varphi}(L)$, then for all $n \geq \max \left(n_{0}, \chi\right)$, $n_{0}=\min \left\{n \in N: d_{1} \varphi\left(\frac{1}{n}\right) \ln n<1\right\}$, the system (3.9) has the unique solution $\left\{\beta_{k}^{*}\right\}_{-n}^{n}$ and the approximate solution,$h_{n}^{*}(t)=\sum_{k=-n}^{n} \beta_{k}^{*} t^{k}$, of equation (2.10) convergences to its exact solution $h^{*}$, moreover $\left\|h^{*}(t)-h_{n}^{*}(t)\right\|_{\varphi} \leq d_{2} \varphi\left(\frac{1}{n}\right) \ln n$,

where $d_{1}$ and $d_{2}$ are constants do not depend on $\mathrm{n}$.

\section{Proof.}

From [8], we can write equation (2.10) in the following form:

setting

$$
h^{+}(\alpha(t))-q(t) h^{-}(t)+\frac{1}{e(t) \pi i} \int_{L} R(t, \tau) h(\tau) d \tau=\frac{g(t)}{e(t)},
$$

$$
q(t)=\frac{\psi^{+}(\alpha(t))}{\psi^{-}(t)}
$$

Then we have

$$
\Gamma h=B h+G h=\tilde{g} .
$$

Where

$$
\begin{aligned}
& (B h)(t)=\psi^{-}(t) h^{+}(\alpha(t))-\psi^{+}(\alpha(t)) h^{-}(t),(G h)(t)=\frac{c(t)}{\pi i} \int_{L} R(t, \tau) h(\tau) d \tau, \\
& \tilde{g}(t)=g(t) c(t), \quad c(t)=\frac{\psi^{-}(t)}{e(t)}, \quad \psi(z)=\exp (\theta(z)),
\end{aligned}
$$

$$
\theta(z)=\frac{1}{\pi i} \int_{L} \frac{\rho(\gamma(\tau))}{\tau-z} d \tau ; z \in D^{+}, \quad \theta(z)=\frac{1}{\pi i} \int_{L} \frac{\rho(\tau)}{\tau-z} d \tau ; z \in D^{-},
$$

where $\gamma(t)$ is the inverse $\alpha(t)$ and $\rho(t)$ is a solution of the Fredholm integral equation of second kind

$$
\rho(t)+\frac{1}{\pi i} \int_{L}\left(\frac{\alpha^{\prime}(\tau)}{\alpha(\tau)-\alpha(t)}-\frac{1}{\tau-t}\right) \rho(\tau) d \tau=\ln q(t) .
$$

Moreover, $B$ is linear and $G$ is completely continuous from $H_{\varphi}(L)$ into itself.

Denote by $X_{n}$ to be the $(2 n+1)$ - dimensional subspace of the space $H_{\varphi}(L)$, and let $Q_{n}$ be the projection operator into the set of interpolation polynomial of degree $n$ with respect to the collocation points $t_{j}, j=\overline{0,2 n}$. Then the system (3.9) can be written in $X_{n}$ as a linear operator

$$
\Gamma_{n} h_{n}=B_{n} h_{n}+G_{n} h_{n}=\tilde{g}_{n},
$$

where 


$$
B_{n} h_{n}=Q_{n} B h_{n}, G_{n} h_{n}=Q_{n} G h_{n}, \quad \tilde{g}_{n}=Q_{n} \tilde{g} .
$$

Now, we determine the difference $\Gamma h_{n}-\Gamma_{n} h_{n} \in X_{n}$, from (3.10),(3.12) we have

$$
\begin{gathered}
\left(\Gamma-\Gamma_{n}\right) h_{n}(t)=\left(I-Q_{n}\right)\left[\left(\psi^{-}(t)-\psi_{n}^{-}(t)\right) h_{n}^{+}(\alpha(t))-\left(\psi^{+}(\alpha(t))-\psi_{n}^{+}(\alpha(t))\right) h_{n}^{-}(t)\right]+ \\
+\left(G-G_{n}\right) h_{n}(t) .
\end{gathered}
$$

where $\psi_{n}$ is polynomial of the best uniform approximation of the function $\psi$ with degree not exceeding $n$.

From $[6,8]$ and inequality (2.7), we have

$$
\begin{gathered}
\left\|h_{n}^{ \pm}\right\|_{\varphi} \leq d_{1}\left\|h_{n}\right\|_{\varphi} \\
\left\|\left[\left(\psi^{-}(t)-\psi_{n}^{-}(t)\right) h_{n}^{+}(\alpha(t))-\left(\psi^{+}(\alpha(t))-\psi_{n}^{+}(\alpha(t))\right) h_{n}^{-}(t)\right]\right\|_{\varphi} \leq \gamma_{0} d_{2} \varphi\left(\frac{1}{n}\right)\|h(t)\|_{\varphi},
\end{gathered}
$$

and

$$
\left\|Q_{n}\right\|_{\varphi} \leq d_{3} \ln n
$$

Hence, we get

$$
\|\left(I-Q_{n}\right)\left[\left(\psi^{-}(t)-\psi_{n}^{-}(t)\right) h_{n}^{+}(\alpha(t))-\left(\psi^{+}(\alpha(t))-\psi_{n}^{+}(\alpha(t))\right) h_{n}^{-}(t)\left\|_{\varphi} \leq d_{4} \varphi\left(\frac{1}{n}\right)(\ln n)\right\| h_{n}(t) \|_{\varphi},\right.
$$

where $d_{4}=\gamma_{0} d_{2} d_{3}$.

Let $J_{n}(t)$ be the polynomial of best uniform approximation to the function

$$
J(t)=\frac{c(t)}{\pi i} \int_{L} R(t, \tau) h_{n}(\tau) d \tau,
$$

Then from [6], we have

$$
\left\|J-J_{n}\right\|_{\varphi} \leq d_{5} \varphi\left(\frac{1}{n}\right)\left\|h_{n}\right\|_{\varphi},
$$

hence for arbitrary $h_{n} \in X_{n}$, we get

$$
\left\|G h_{n}-G_{n} h_{n}\right\|_{\varphi} \leq d_{6} \varphi\left(\frac{1}{n}\right)(\ln n)\left\|h_{n}\right\|_{\varphi},
$$

where $d_{6} \ln n=d_{5}+d_{3} d_{5} \ln n$. From (3.13)- (3.15), we get

$$
\left\|\Gamma h_{n}-\Gamma_{n} h_{n}\right\|_{\varphi} \leq d_{7} \varphi\left(\frac{1}{n}\right)(\ln n)\left\|h_{n}\right\|_{\varphi},
$$

where $d_{7}=d_{4}+d_{6}$. From Theorem 2.2 , the operator $\Gamma_{0}$ has a linear bounded inverse operator $\Gamma_{0}^{-1}$, since $\Gamma_{0} h=c^{-1} \Gamma h$ then the operator $\Gamma$ has a linear inverse, also from [6] and by virtue of (3.16) the operator $\Gamma_{n}$ has a linear bounded inverse.

Now, for the right parts of (3.10) and (3.12), we have

$$
\left\|\tilde{g}-\tilde{g}_{n}\right\|_{\varphi} \leq d_{8} \varphi\left(\frac{1}{n}\right) \ln n \text {. }
$$


From [6], and inequalities (3.16), (3.17) for the solution $h^{*}$ of equation (2.10) and the approximate solution $h_{n}^{*}$, we obtain

$$
\left\|h^{*}-h_{n}^{*}\right\|_{\varphi} \leq d_{9} \varphi\left(\frac{1}{n}\right) \ln n .
$$

Thus the theorem is proved.

From Theorem 3.1 there exists the number $n_{0}$ such that for arbitrary $n \geq \max \left(n_{0}, \chi\right)$ the SLAES (3.6) has the unique solution $h^{*}$ and the following inequality is valid:

$$
\left\|u_{n}^{*}\left(h^{*}, .\right)-u^{*}(.)\right\|_{\varphi} \leq d_{10} \varphi\left(\frac{1}{n}\right) \ln n,
$$

Where $u^{*} \in H_{\varphi}(L)$ is the unique solution of (3.7). Let

$$
\Gamma_{n}\left(u_{0}\right) h=\left(\Gamma_{0, n}\left(u_{0}\right) h, \ldots, \Gamma_{2 n, n}\left(u_{0}\right) h\right)
$$

where

$$
\begin{aligned}
\left.\Gamma_{n, j}\left(u_{0}\right) h=a\left(t_{j}\right) u_{n}\left(h, t_{j}\right)\right)+b\left(t_{j}\right) u_{n}\left(h, \alpha\left(t_{j}\right)\right)-\frac{a\left(t_{j}\right)}{\pi i} \int_{L} \frac{u_{n}(h, \tau)}{\tau-t_{j}} d \tau+ \\
+\frac{b\left(t_{j}\right)}{\pi i} \int_{L} \frac{u_{n}(h, \tau)}{\tau-\alpha\left(t_{j}\right)} d \tau+\frac{1}{\pi i} \int_{L} R\left(t_{j}, \tau\right) u_{n}(h, \tau) d \tau, j=\overline{0,2 n}
\end{aligned}
$$

From [6], we have

$$
\left\|\Gamma_{n}\left(u_{0}\right)-\mathrm{P}_{n}^{\prime}\left(\eta^{(0)}\right)\right\|_{H_{\varphi}^{(1)} \rightarrow H_{\varphi}^{(2)}} \leq d_{11} \varphi\left(\frac{1}{n}\right) \ln n
$$

Since for arbitrary $n \geq\left(n_{0}, \chi\right)$, there exists a bounded linear inverse operator, $\Gamma_{n}^{-1}: H_{\varphi}^{(2)} \rightarrow H_{\varphi}^{(1)}$ then from (3.18), Banach theorem follows that there exists $n_{1} \geq\left(n_{0}, \chi\right)$ such that for arbitrary $n \geq n_{1}$, the linear operator $\mathrm{P}_{j, n}^{\prime}$ has bounded inverse, that is the SLAES (3.4) under condition (3.5) has the unique solution $h^{*} \in H_{\varphi}^{(1)}$ for arbitrary right side $g=g\left(t_{j}\right) \in H_{\varphi}^{(2)}, j=\overline{0,2 n}$. Thus the following theorem is proved.

Theorem 3.2 Let the coordinate of the vector $\eta^{(0)}=\left(\eta_{-n}^{(0)}, \ldots, \eta_{-1}^{(0)}, \eta_{0}^{(0)}, \ldots, \eta_{n}^{(0)}\right)$ be the Fourier coefficients the function $u_{0} \in H_{\varphi}(L)$ and the conditions of Theorem 1.2 are satisfied and for $n \geq n_{1},\left\|\left(\mathrm{P}_{n}^{\prime}\left(\eta^{(0)}\right)\right)^{-1}\right\|_{\varphi} \leq \varepsilon_{0}^{\prime}$ and $\left\|\left(\mathrm{P}_{n}^{\prime}\left(\eta^{(0)}\right)\right)^{-1} \mathrm{P}_{n}\left(\eta^{(0)}\right)\right\|_{\varphi} \leq \varepsilon_{1}^{\prime}$. Then if $m^{\prime}=\varepsilon_{0}^{\prime} \rho_{1}^{\prime} \varepsilon_{1}^{\prime}<1 / 2$, then SNAES (3.3) has the unique solution $\eta^{*}=\left(\eta_{-n}^{*}, \ldots, \eta_{-1}^{*}, \eta_{0}^{*}, \ldots, \eta_{n}^{*}\right)$ in the sphere $S_{\varphi}\left(\eta^{(0)} ; r_{0}^{\prime}\right)$ of the space $H_{\varphi}(L), r_{0}^{\prime}=\varepsilon_{1}^{\prime}\left(1-\sqrt{1-2 m^{\prime}}\right)\left(m^{\prime}\right)^{-1} \leq r^{\prime}$, to which the following iteration process converges $\eta^{(m+1)}=\eta^{(m)}-\left(\mathrm{P}_{n}^{\prime}\left(\eta^{(0)}\right)\right)^{-1} \mathrm{P}_{n}\left(\eta^{(m)}\right)$ and the rate of convergence is given by the inequality: $\left\|\eta^{(m)}-\eta^{*}\right\|_{\varphi} \leq \frac{B_{1}^{n}}{1-B_{1}} \varepsilon_{1}^{\prime} ; B_{1}=1-\sqrt{1-2 m^{\prime}}$. 


\section{REFERENCES}

1. S. M. Amer and S. Dardery, The Method of Kantorovich Majorants to Nonlinear Singular Integral Equation with Shift, Appl. Math. and Comp. 215: 2799-2805, 2009.

2. S. M. Amer and S. Dardery, On the Theory of Nonlinear Singular Integral Equations with Shift in Holder Spaces, Forum Math. 17: 753-780, 2005.

3. S. M. Amer and S. Dardery, On a Class of Nonlinear Singular Integral Equations with Shift on a Closed Contour, Appl. Math. And Comp. 158: 781-791, 2004.

4. S. M. Amer and A. S. Nagdy, On the modified Newton's approximation method for the solution of nonlinear singular integral equations; Hokkaido Mathematical Journal. 29 59-72, 2000.

5. S. M. Amer, On Solution of Nonlinear Singular Integral Equations with Shift in Generalized Holder Space; Chaos, Solitons and Fractals. 12: 1323-1334, 2001.

6. S. M. Amer, On the approximate Solution of Nonlinear Singular Integral Equations with Positive Index, Int. J. Math. Math. Sci. 19(2): 389-396, 1996.

7. A. A. Baturev, V. G. Kravchenko and G. S. Litvinchuk, Approximate Method for Singular Integral Equation with a Non-Carleman Shift; Integral Equation App. 8: $1-17,1996$.

8. F. D. Gakhov, Boundary Value Problems; English Edition Pergamon Press Ltd, 1966.

9. A. I. Guseinov and Kh. Sh. Mukhtarov, Introduction to the Theory of Nonlinear Singular Integral Equations, Nauka Moscow (in Russian), 1980.

10. D. Jinyuan, The Collocation Methods and Singular Integral Equations with Cauchy Kernel, Acta Math. Sci. 20 (B3): 289-302, 2000.

11. P. Junghanns and U. Weber, On the Solvability of Nonlinear Singular Integral Equations, ZAA.12: 683-698, 1993.

12. L.V. Kantorovich, G. P. Akilov, Functional Analysis; Pergamon Press. Oxford, 1982.

13. K V.G.ravchenko, A.B. Lebre, G.S. Litvinchuk and F.S. Texeira, Fredholm Theory for a Class of Singular Integral Operators with Carleman Shift and Unbounded Coefficients, Math. Nach. 172 : 199-210, 1995.

14. V.G. Kravchenko and G.S. Litvinchuk, Introduction to the Theory of Singular Integral Operators with Shift; Kluwer Academic Publishers, 1994.

15. E.G. Ladopoulous, V.A. Zisis, Nonlinear Singular Integral Approximations in Banach Spaces, Nonlinear Analysis, Theory, Methods Appl. 26(7): 1293-1299, 1996.

16. E.G. Ladopoulous, Singular Integral Equations Linear and Non-linear Theory and its Applications in Science and Engineering; Berlin, Springer, 2000.

17. G. S. Litvinchuk, Boundary Value Problems and Singular Integral Equations with Shift; Nauka, Moscow, (in Russian), 1977.

18. S. G. Mikhlin and S. Prossdorf, Singular Integral Operator; Academy-Verleg, Berlin, 1986. 
19. D. T. Nguyen, On a Class of Nonlinear Singular Integral Equations with Shift on Complex Curves; Acta Math. Vietnam. 14 (2): 75- 92, 1989.

20. W. Pogorzelski, Integral Equations and their Applications; Oxford Pergamon Press and Warszawa, PWN, 1966.

21. M.H. Saleh and S.M. Amer, On a class of Nonlinear Singular Integral Equations with Cauchy Kernel; AMSE Review. 22 (1): 15-26, 1992.

22. N.P. Vekua, Systems of Singular Integral Equations, Noordhoff Ltd, Groningen, 1967.

23. L. V. Wolfersdorf, On the Theory of Nonlinear Singular Integral Equations ; TU Bergakde-min Freiberg, Fakultat fur Mathematik und Informatik; lecture presented on the international symposium "Operator Equations and Numerical Analysis", September 28-October 2, 1992.

24. L. V. Wolfersdorf, On the Theory of Nonlinear Singular Integral Equations of Cauchy Type; Math. Meth. In the Appl. Sci. 7: 493-517, 1985. 